\title{
Biomagnetism and Prostate
}

\author{
Anninos P*, Papadopoulos I, Adamopoulos A and Kotini A \\ Lab of Medical Physics and Dept of Urology, Medical School, Democritus University of \\ Thrace, Alexandroupolis, Greece
}

*Corresponding author: Emeritus Professor P Anninos, Lab of Medical Physics,

\section{Editorial}

Volume 3 Issue 3

Received Date: March 12, 2018

Published Date: March 19, 2018

Medical School, Democritus University of Thrace, University Campus, 68100, Alexandroupolis, Greece, Tel: +3025510 30392; E-mail: pans.photios.anninos@gmail.com

\section{Editorial}

Prostate cancer is a dangerous disease that is frequently identify at a late stage and is accompany by high mortality. Novel techniques are necessary in order to identify it at an early stage. Biomagnetometer is a technique that has been successfully used in differentiating breast, ovarian lesions and myomas [1,2]. We have investigated the function of bio magnetic activity in distinguishing cancerous from benign prostate lesions. The prostate gland, as every alive tissue, produces spontaneous magnetic activity caused by ionic movements across the plasma membrane. This activity, though extremely weak can be recorded by a superconducting quantum interference device (SQUID).

We used a single channel second order biomagnetometer (DC SQUID mode1 601, Biomagnetic Technologies) in an electrically shielded room. The data were achieved from 47 patients with palpable prostate lesions. Twenty-four had prostate carcinoma and 23 benign prostatic hyperplasia $(\mathrm{BPH})$. Every patient lied supine on a wooden bed, with no metallic objects. Biomagnetic data of the prostate activity were carried out with a detector placed on the abdomen above the symphysis pubis. We selected 8 points for examination (4 located at the very centre of the prostate lesion, and 4 at the periphery of the examined part). For every point, 32 sec duration recordings were taken with the SQUID detector placed $3 \mathrm{~mm}$ above each point in order the maximum magnetic flux to pass through the coil. All recordings were done prior to histological examination and the operators were blinded to medical and transrectal ultrasound findings $[3,4]$.
The biomagnetic activity of malignant tissues is a indication of tumour hyperemia and neo-vascularisation and is caused by the movement of ions across the plasma membrane. The activity of the prostatic lesions were of high amplitude in the majority of carcinomas and of low amplitude in most BPH patients. In every case, the frequencies considered were distributed within the range of 2-7 Hz. The corresponding spectral densities of the magnetic field were of high amplitude in the malignant prostatic neoplasms and of low amplitude in benign prostatic lesions.

We also investigated the complexity underlying the dynamics which characterized the benign and malignant prostate lesions, using chaotic analysis. By applying the Grassberger-Procaccia algorithm to the diamagnetic data we found clear saturation in malignant prostate lesions and not clear saturation in benign ones. This substantiates an elevated angiogenic activity in prostate cancer than in $\mathrm{BPH}$ lesions. When we estimated the correlation dimension, we found a saturation value for the cancer lesions that demonstrates a lower complexity of the system in compare to the $\mathrm{BPH}$, which is characterized by higher complexity $[3,4]$.

Malignant tissues, by virtue of their expansion and increased ionic movements, produce magnetic fields of higher intensity than benign ones. In our studies the biomagnetic activity of the prostatic carcinomas was found lower compared to breast carcinomas. These results could be explained by the lower angiogenic activity of prostate carcinomas, in compare to other malignancies $[3,4]$. 


\section{Psychology \& Psychological Research International Journal}

Biomagnetic activity of prostate lesions might prove a valuable means in detecting prostatic carcinomas and might suggest extra information for understanding better the biology of prostate cancer. The technique is noninvasive, harmless, consistent, fast and simple to interpret with a disadvantage the quite big distance between the suprapubic biomagnetic detector and the centre of the prostate gland $(3-5 \mathrm{~cm})$. However, the exact function of biomagnetic activity in prostate cancer detection and biology remains to be additional investigated with extra studies to be conducted $[3,4]$.

\section{References}

1. Anninos P, Anastasiadis P, Adamopoulos A, Kotini A (2016) Biomagnetic activity and non linear analysis in obstetrics and gynecology in a Greek population. Clin Exp Obstet Gynecol 43(3): 406-408.

2. Kotini A, Anastasiadis AN, Koutlaki N, Tamiolakis D, Anninos P, et al. (2006) Biomagnetism in gynaecologic oncology. Our experience in Greece. Eur J Gynaecol Oncol 27(6): 594-596.

3. Anninos P, Papadopoulos I, Kotini A (2008) Biomagnetic activity in prostate cancer and BPH. Arch Esp Urol 61(3): 459-463.

4. Anninos P, Papadopoulos I, Kotini A, Adamopoulos A (2003) Differential diagnosis of prostate lesions with the use of biomagnetic measurements and non-linear analysis. Urol Res 31(1): 32-36. 